\title{
Zauberberg en Miniature im Spielzeugmuseum Davos
}

\author{
A Miniature "Magic Mountain" in the Toy Museum Davos
}

Autoren

Institute
R. Kropp ${ }^{1}$, T. Nelson ${ }^{2}$

1 Petersberg

2 Leiter der Dokumentationsbibliothek Davos, Davos, Schweiz
Bibliografie

Dol http://dx.doi.org/ 10.1055/s-0030-1256073 Pneumologie 2011; 65: 21-24 @ Georg Thieme Verlag KG Stuttgart · New York ISSN 0934-8387

\section{Korrespondenzadresse} Dr. med. Robert Kropp Liegnitzer Straße 5 36100 Petersberg dr.robert.kropp@gmx.de
Zu den Orten in der Welt, deren Namen fest mit der Tuberkulose verbunden sind, gehört sicherlich Davos in Graubünden. Spaziert man dort auf der „Promenade“ von Davos Dorf in Richtung Davos Platz, findet man rechter Hand einige Häuser nach dem Hotel Steigenberger Belvédère das private Spielzeugmuseum Angela Prader Davos. Seit 1996 wurde es vom Ehepaar Angela und Jürg Prader aufgebaut und betreut; zur Museums-Philosophie äußerten sich die Besitzer wie folgt: „Das Museum soll nicht nur seltene Exponate zeigen, sondern auch die handwerkliche und künstlerische Meisterschaft der damaligen Hersteller vor Augen führen. Entsprechend war die Welt der Spielzeuge früher eine andere Welt als wir sie heute kennen. Wir sehen es als unsere Aufgabe, die frühere Spielzeugwelt auch für spätere Generationen zu erhalten."

Beginnend mit einer Ausstellung interessanter Puppen ist das Museum im Laufe der Jahre zu einer der vielseitigsten und größten Sammlungen Europas gewachsen; es enthält einen umfangreichen Fundus verschiedenartigster kunsthistorisch bedeutender Spielsachen und technischer Spielzeuge des 18.-20. Jahrhunderts in originalem Zustand, welche in den drei Stockwerken des Hauses als Dauer-Ausstellung in 90 Vitrinen auf $600 \mathrm{~m}^{2}$ exemplarisch zu besichtigen sind. Darüber hinaus werden immer wieder Sonderausstellungen zu unterschiedlichen Themen angeboten $(\checkmark$ Tab. 1). Eine besucherfreundliche Ausstellungstechnik und gute Beleuchtung erhöhen das Vergnügen der Besichtigung.

Unter den sehr sehenswerten Exponaten der Spielzeugkunst und Spielzeugkultur, seit 1730 bis um 1960, darunter seltene oder sogar einmalige Ausstellungsstücke, sticht als besondere Attraktion dieses international hoch eingestuften Museums ein großes Schaumodell eines Tuberkulose-Sanatoriums hervor ( $\bullet$ Abb. 1).

Dieses zauberhafte Exponat ist, wie Frau Prader erzählt, ein handgemachtes Unikat, welches die

\begin{tabular}{|c|c|}
\hline $2010 / 2011$ & $\begin{array}{l}\text { „Taschen, Täschchen - vergessene } \\
\text { Kostbarkeiten“ }\end{array}$ \\
\hline $2009 / 2010$ & "Welt der Spielzeugbahnhöfe“ \\
\hline $2008 / 2009$ & $\begin{array}{l}\text { "Arche Noah - Menschen, Boot und } \\
\text { Tiere“ }\end{array}$ \\
\hline $2007 / 2008$ & $\begin{array}{l}\text { "Barbie - Mädchentraum und Kult- } \\
\text { figur“ }\end{array}$ \\
\hline $2006 / 2007$ & $\begin{array}{l}\text { "Die schrägen 50er Jahre - Wohn- } \\
\text { kultur im Puppenhaus“" }\end{array}$ \\
\hline $2005 / 2006$ & „Das Rote Kreuz im Spielzimmer“ \\
\hline $2004 / 2005$ & $\begin{array}{l}\text { „MECCANO Konstruktionen mit } \\
\text { Metallbaukästen“ }\end{array}$ \\
\hline $2003 / 2004$ & $\begin{array}{l}\text { „Spielzeugeisenbahnen nach } \\
\text { Schweizer Vorbild“ }\end{array}$ \\
\hline
\end{tabular}

Stimmung in einem damaligen Tuberkulose-Sanatorium vermitteln soll. Die $\bullet$ Abb. 2-4 zeigen Ausschnitte des Modells, ein Krankenzimmer, einen Ordinationsraum für den Arzt und ein Speisezimmer.

Das Modell ist dem Sanatorium Berghof aus dem Roman Der Zauberberg ${ }^{1}$ (1924) von Thomas Mann nachempfunden. Architektonisch entspricht es in etwa dem früheren Internationalen Sanatorium Dr. Philippi bzw. Sanatorium Valbella in Davos Dorf ( $\bullet$ Abb. 5 u. 6). (Infolge der Internierung von Hunderten deutscher Militärpatienten in Davos im Ersten Weltkrieg wurde das Sanatorium nochmals umbenannt. Ab 1918 hieß es dann Deutsches Kriegerkurhaus. Das Gebäude steht noch, allerdings ist die Fassade durch Umbau stark verändert.) Die Inneneinrichtung des Modells ist historisch nachgebildet, die Figuren und alles Zubehör stammen aus der Zeit des Romans. Hinter dem Modell steht ein großes Talent. Diese Person ließ sich eindeutig von der Beschreibung des Sanatoriums im „Zauberberg“ inspirieren, sowohl für

\footnotetext{
${ }^{1}$ Das Bild auf dem Umschlag der neuesten Taschenbuchausgabe zeigt das Sanatorium Valbella und stammt aus der Sammlung der Dokumentationsbibliothek Davos (DBD).
} 


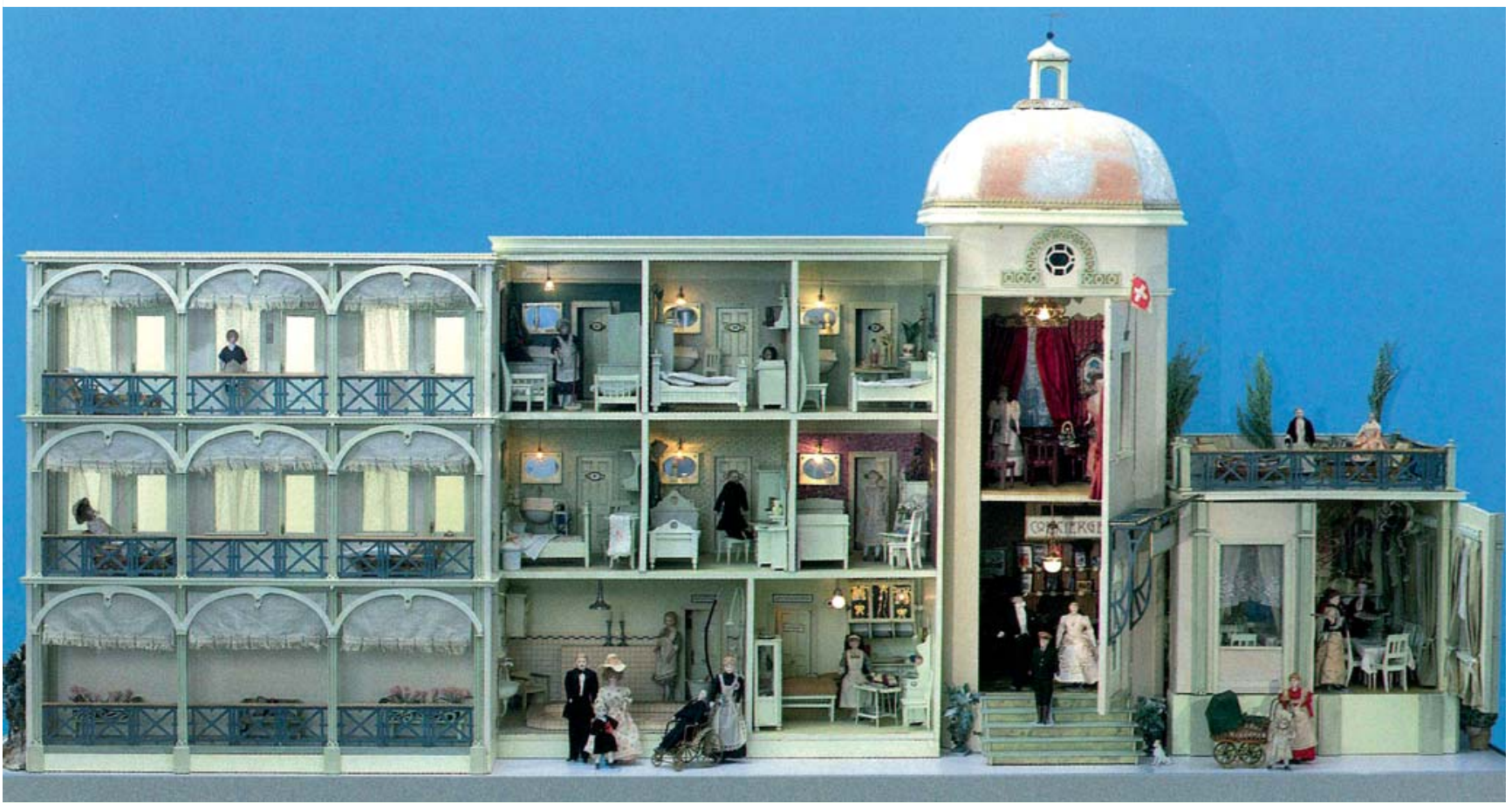

Abb. 1 Schaumodell, Übersicht.

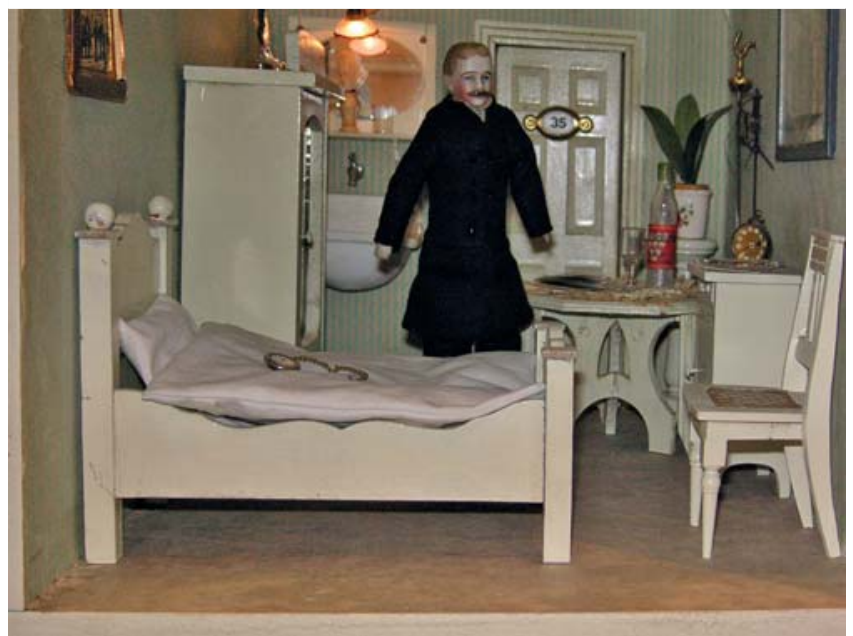

Abb. 2 Schaumodell, Krankenzimmer.

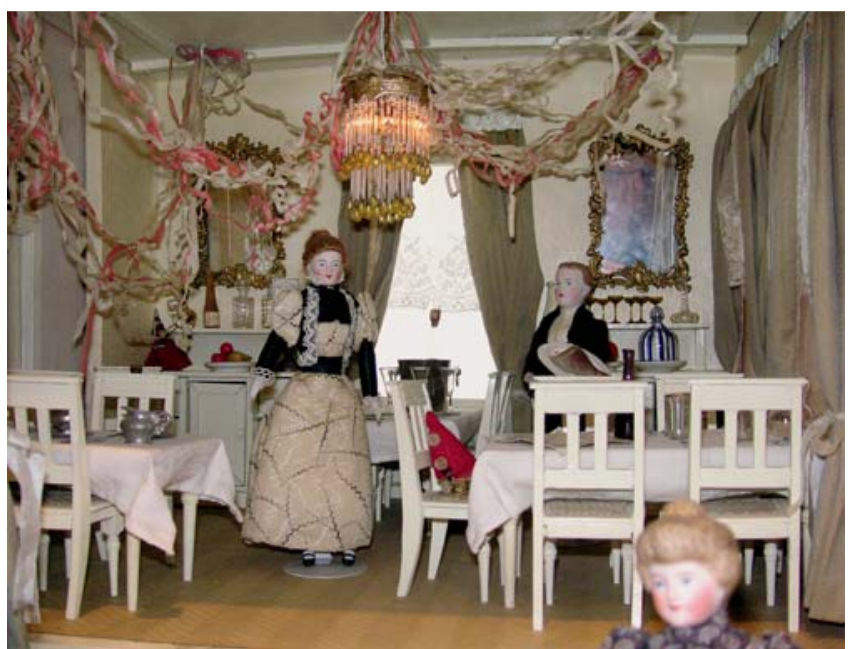

Abb. 4 Schaumodell, Speisezimmer.

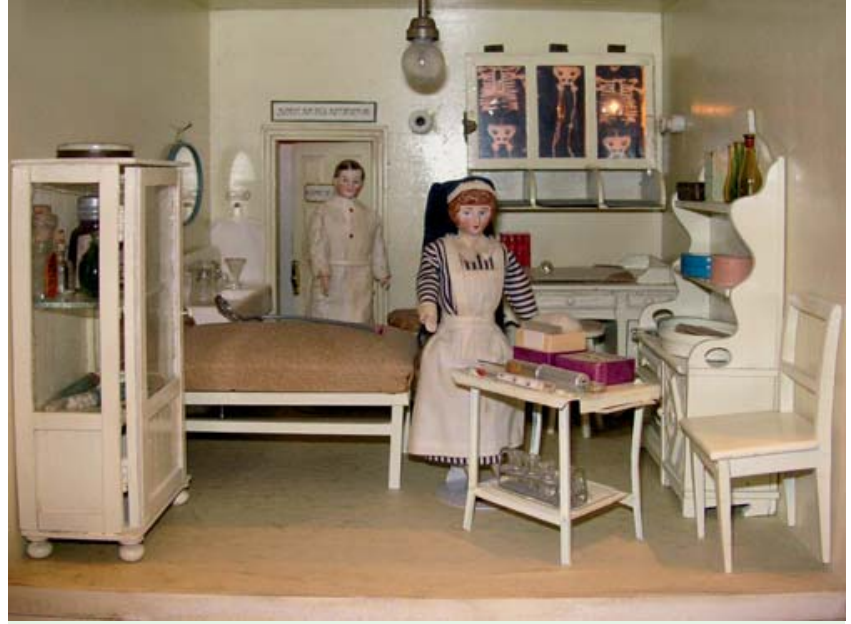

Abb. 3 Schaumodell, Ordinationsraum.

das Exterieur (vgl. Kuppel) wie auch für diverse Details des Interieurs (vgl. Nummernschild an der Tür des Krankenzimmers).

Welches Sanatorium aber stand Pate bei der Arbeit Thomas Manns an seinem Roman? Die Frage lässt sich mit einem Namen nicht beantworten. Bei seiner Schilderung des Alltags im Sanatorium Berghof hat sich der Künstler große Freiheiten erlaubt, und einen Berghof gab es in Davos genauso wenig wie einen Berg namens Zauberberg. Dennoch ist manches im Roman durchaus „historisch“. Über einen Mangel an Stoff für einen Sanatoriumsroman konnte sich der Verfasser an diesem Ort wahrlich nicht beschweren! Erinnern wir uns: Davos wurde in der Zeit der Belle Époque zu einer ordentlichen, kosmopolitischen Stadt. 1910 lebten fast 10000 Menschen hier (1870 waren es knapp 2000), es gab 26 Sanatorien, 34 Hotels und 51 Pensionen. (In einigen Hotels und Pensionen kamen auch Patienten unter.) Laut der wöchentlichen Statistik fluktuierte in der Zeit unmittelbar vor dem Ersten Weltkrieg die Zahl der anwesenden Patienten und Touristen, je nach Saison, zwischen 3000 und 5500. Die Gäste - z. T. sehr wohl- 


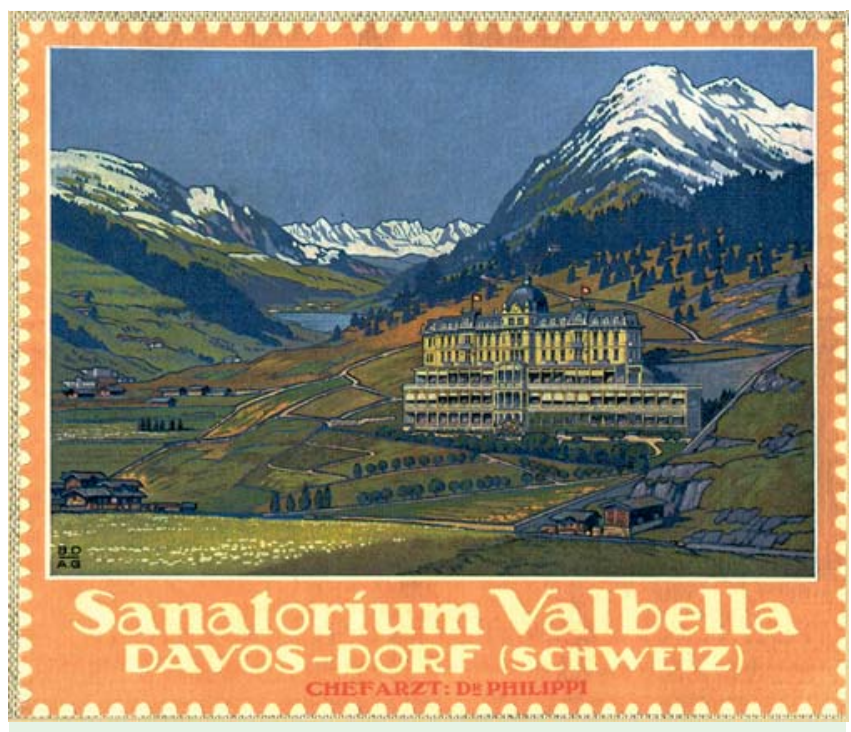

Abb. 5 Sanatorium Valbella, Davos Dorf. Aus einem Prospekt 19151916. habend - kamen aus aller Herren Ländern, entsprechend war auch das Bedürfnis nach Zerstreuung und Information. Fast allwöchentlich erschien eine Kurortszeitschrift in vier Sprachen, alle als Separatdrucke erhältlich (Deutsch, Englisch, Französisch und Russisch). Zu jeder Ausgabe gab es eine „Fremden-Liste“ (12 bis mehr als 20 Seiten!) mit Namen und Herkunftsort der Gäste. Thomas Mann besuchte seine Frau Katia im Frühling 1912, als sie im Waldsanatorium Prof. Jessen (heute Waldhotel Bellevue), Davos Platz, weilte ( $\bullet$ Abb. 7 u. 8). Belegt ist „Frau Thomas Mann, München“ in den „Fremden-Listen“ seit dem 22. März. „Herr Thomas Mann, München“ wird als Gast in den Listen von 18. und 31. Mai, und vom 8. Juni registriert $(\bullet \text { Abb. } 9 \text { u. } \mathbf{1 0})^{2}$.

Im Waldsanatorium - und an anderen Orten - sammelte er Material für seinen Roman. Das Innenleben einer Heilstätte konnte er hier genau beobachten, und die Inneneinrichtungen dieses Hauses dienten als Vorlage für seine Arbeit. Von außen gesehen aber diente dem Verfasser, wie bereits erwähnt, das Sanatorium Valbella in Davos Dorf und nicht das Waldsanatorium oberhalb der „Promenade“ am Platz. (Die dritte, „historische“ Davoser Heilstätte, Sanatorium Schatzalp, das ja im Roman beim richtigen Namen genannt wird, scheint weniger wichtig zu sein als An-

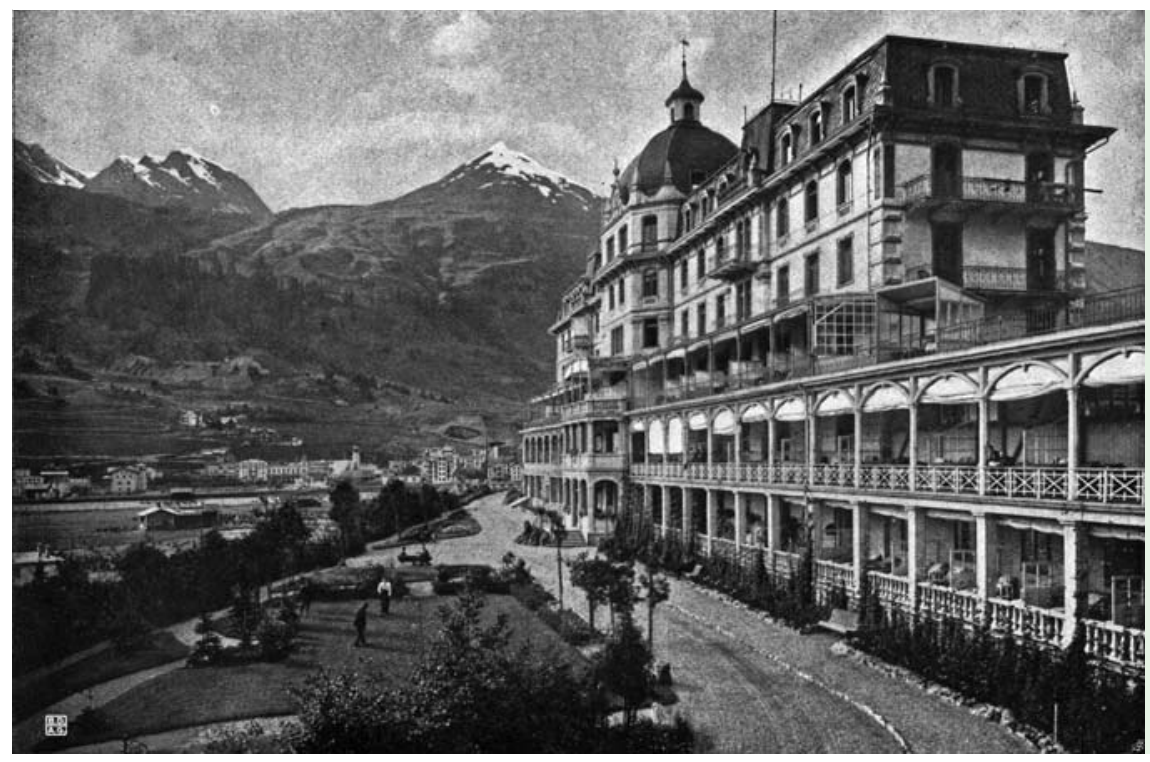

Abb. 6 Sanatorium Valbella, Davos Dorf, Fotografie 1915-1916 (E. Himmelsbach).
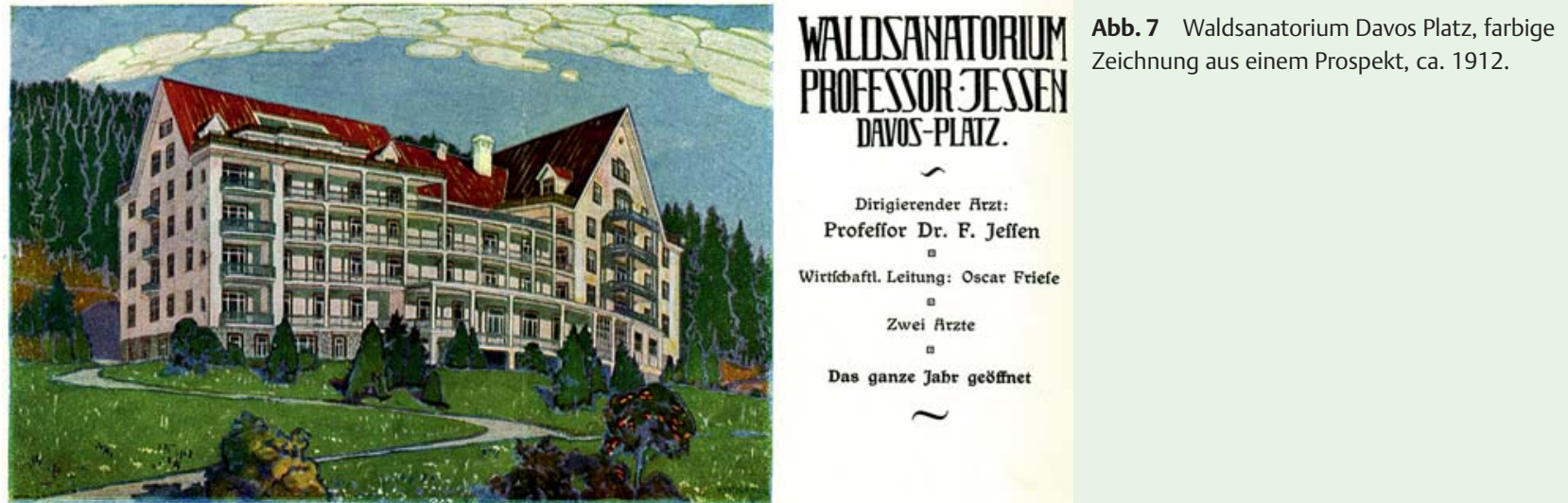
Dirigierender Hrzt:
Profeffor Dr. F. Jeffen
Wirtichaftl. Leitung: Oscar Friefe
Zwei Arzte
(

Das ganze Jabr geơffnet

$\sim$

\footnotetext{
2 „Frau Thomas Mann, München“ und „Herr Thomas Mann, München“ sind in der Fremdenliste vom 31. Mai 1912 auf der Seite 2, in der mittleren Spalte, Waldsanatorium Professor Jessen, gelistet.
} 


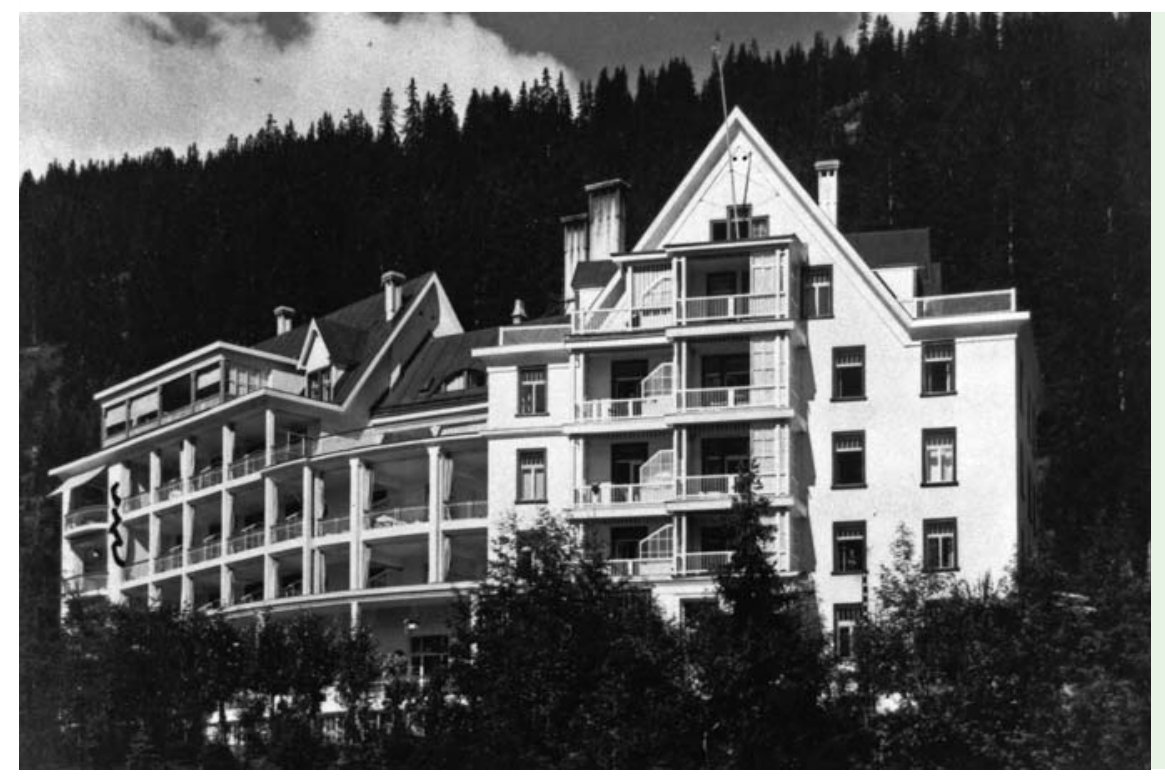

Abb. 8 Waldsanatorium Davos Platz, Fotografie 1920 (E. Meerkämper).

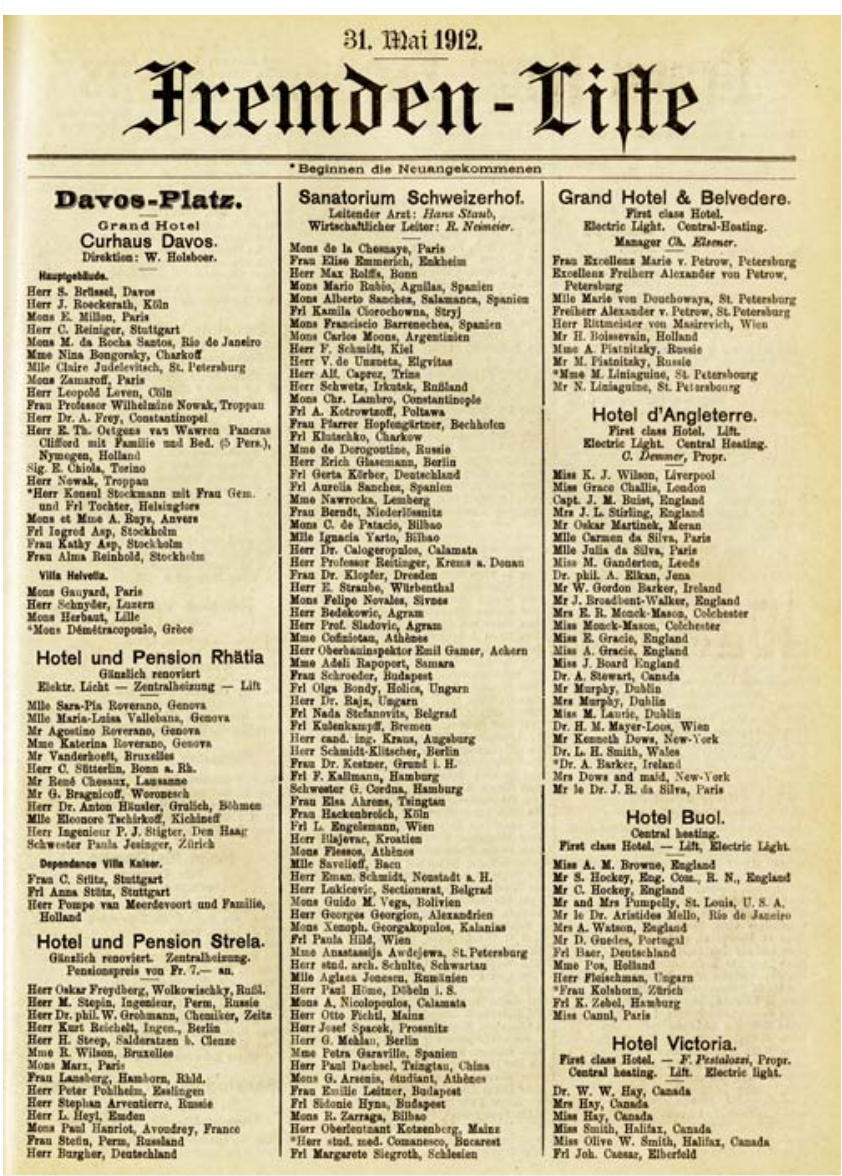

Abb. 9 Fremdenliste vom 31. Mai 1912, Titelblatt.

regung für die Schilderung von Räumlichkeiten und Architektur im Roman.)

Wer den literarischen Text von Thomas Mann kennt, wird im liebevoll hergerichteten Modell des fiktiven Sanatoriums Berghof im Spielzeugmuseum Davos Bezüge zum Roman - und auch zur tatsächlichen Sanatoriumsgeschichte - auf Schritt und Tritt entdecken können.

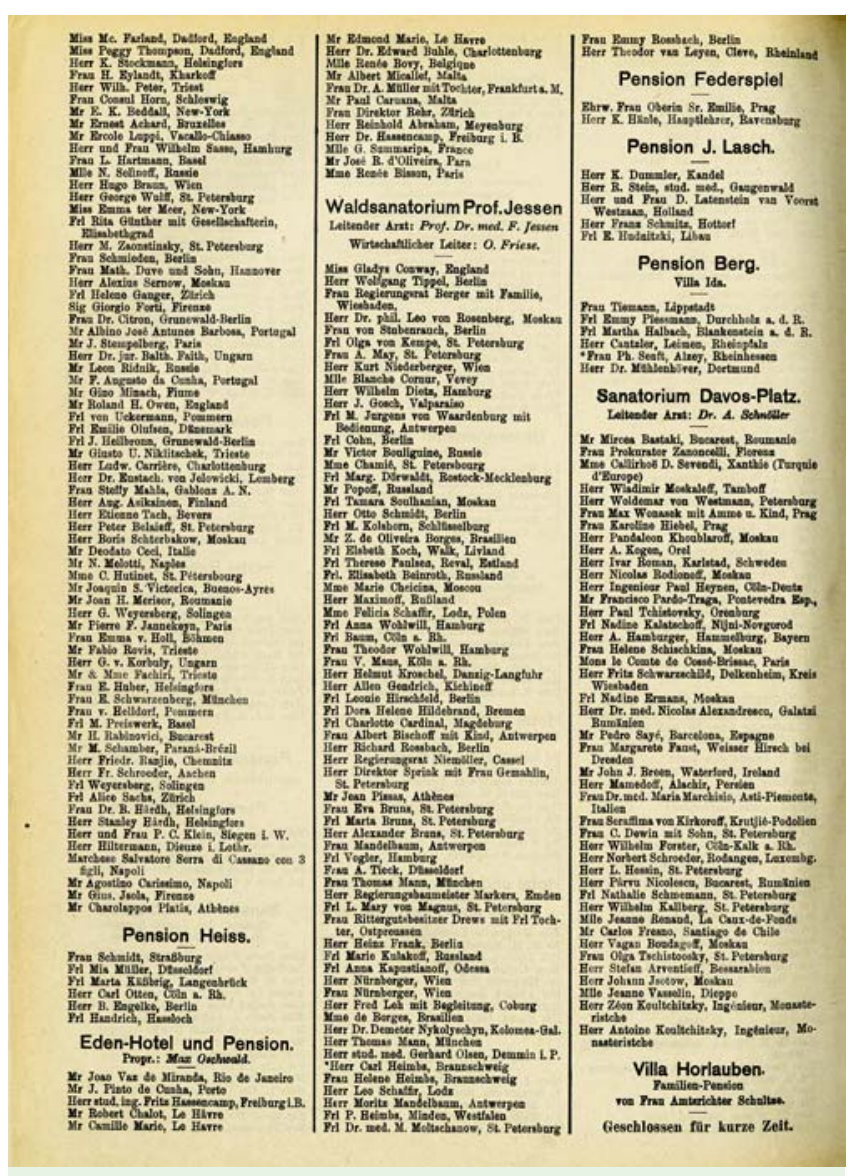

Abb. 10 Fremdenliste vom 31. Mai 1912, Seite 2.

\section{Verwendete Literatur}

1 Davoser Blätter, Jg. 41 (1912). Verschiedene Ausgaben von März - Juni

2 Davoser Revue, Jg. 69, Nr. 3 (August 1994). Sondernummer „Der Zauberberg“"

3 Mann T. Der Zauberberg. Frankfurt/M: S. Fischer; 2008

4 Rott M. Zauberberg-Wanderung. Vom Waldhotel Davos zum ThomasMann-Platz auf der Schatzalp. Würzburg: Königshausen \& Neumann; 2009

5 Sprecher T. Davos im Zauberberg. Zürich: Neue Zürcher Zeitung; 1996 